\title{
Reply to the brief communication "Essential review points on the retrospective study of osteoradionecrosis in the jaws"
}

\author{
Brena Rodrigues Manzano, Paulo Sérgio da Silva Santos \\ Department of Surgery, Stomatology, Pathology and Radiology, Bauru School of Dentistry, University of São Paulo, Bauru, Brazil
}

\begin{abstract}
To the Editor,
The brief communication from Seo and $\mathrm{Kin}^{1}$ addressed some questions about our original article titled "Retrospective study of osteoradionecrosis in the jaws of patients with head and neck cancer"2 . Initially, a retrospective study was planned from a specialized center, on the multidisciplinary supportive care of cancer patients directed by experienced professionals in the care of this profile of patients. Most medical information was obtained through good relationships with responsible radiotherapists. In addition, the specialized center has at its disposal, equipment for panoramic radiography and cone beam computed tomography, providing a more accurate and complete diagnosis of the complications of radiotherapy treatment, such as osteoradionecrosis (ORN). It is the protocol at our specialized center to perform imaging during the initial evaluation and monitoring of all patients undergoing any type and dose of radiotherapy in the head and neck, such as cobalt, and intensity-modulated radiation therapy (IMRT) for ORN risks.
\end{abstract}

To the best of our knowledge, we could not find any paper to define conservative treatment for ORN. In our original article, curettage, irrigation with $0.12 \%$ chlorhexidine, antibiotic use, bone fragment removal, hyperbaric therapy, and use of pentoxifylline and tocopherol (PENTO) was considered conservative treatment versus ostectomy and mandibulectomy, which are considered more aggressive, in other studies ${ }^{3}$.

\footnotetext{
Paulo Sérgio da Silva Santos

Department of Surgery, Stomatology, Pathology and Radiology, Bauru School of Dentistry, University of São Paulo, Alameda Doutor Octavio Pinheiro Brisolla, 9-75, Vila Universitária, Bauru 17012-901, São Paulo, Brazil

TEL: +55-14-3226-6113 FAX: +55-14-3235-8258

E-mail:paulosss@fob.usp.br

ORCID: https://orcid.org/0000-0002-0674-3759

(c) This is an open-access article distributed under the terms of the Creative Commons Attribution Non-Commercial License (http://creativecommons.org/ licenses/by-nc/4.0/), which permits unrestricted non-commercial use, distribution, and reproduction in any medium, provided the original work is properly cited.

Copyright (C) 2020 The Korean Association of Oral and Maxillofacial Surgeons. All rights reserved.
}

The use of PENTO seems to be a promising alternative, but all systematic reviews and meta-analysis show the need for further prospective clinical trials using PENTO in the treatment of ORN; therefore, the evidence remains insufficient to determine its treatment protocol for $\mathrm{ORN}^{3}$. The study cited by the authors used PENTO as a treatment for diseases with different etiopathogenesis as ORN and osteonecrosis of the jaws related to medications ${ }^{4}$. We do not understand how it is not possible to analyze the results of PENTO treatment of bone necrosis of different diseases included in the same group. In addition, in our study, only one patient used PENTO without ORN resolution ${ }^{2}$.

The authors, Seo and $\mathrm{Kin}^{1}$ questioned the presence of ORN only in stages II and III in our sample. Most patients with ORN have been referred from other centers, that is, they have already attended our center with a diagnosis of ORN and all patients in our center are followed up before, during, and after radiotherapy treatment with prevention protocols. Therefore, we agree that most of the cases presented in this study present ORN in the more advanced stages. This may be due to the limitations of retrospective studies, as discussed in the original article (fifth paragraph of the discussion) ${ }^{2}$. The acute and late effects of ORN are well established in literature, but in this study, we evaluated ORN exclusively.

The authors, Seo and $\mathrm{Kin}^{1}$ reported in the brief communication that there is no time between ORN and radiotherapy; however, this has been described in the eighth column of Table 1 (beginning of ORN) as well as the data on clinical progress of ORN (last column of Table 1), thereby suggesting careful reading of our original article ${ }^{2}$.

The treatment of ORN is multimodal, but the more conservative the treatment in these patients, the better their quality of life, including treatment with PENTO as one of the conservative treatment options. Obtaining good results when performing conservative treatment of ORN in stage III cases is an indicator of success. Some patients are clinically 
unstable to undergo major surgical interventions, and conservative treatment becomes an alternative for the control of ORN infection in these patients, by decreasing morbidity and improving the quality of life.

The errors in Tables 2, 3, and 4 have been corrected and annexed. In Table 2, there was an overlap of 2 individuals included in "others", which ranged from $12(8.6 \%)$ to $10(7.1 \%)$. In Table 3, 20 other oral cancer patients and 30 other patients with tumors in the head and neck did not present ORN, and they were added to a total of 139 patients. In Table 4, the incidence of ORN according to each antineoplastic treatment modality in 139 patients, was 10/60 (60.0\%) radiotherapy (RT), 6/32 (18.8\%) RT+chemotherapy (CT)+surgery, 4/30 (13.3\%) $\mathrm{RT}+$ surgery, and 4/67 (5.9\%) RT+CT. The changes in all tables did not change the order of incidence of the factors; thus, the changes clarify the mathematical doubts of readers without invalidating our study. The revision of the Tables can be found in the Corrigendum, which is also published in this issue (https://doi.org/10.5125/jkaoms.2020.46.6.443).

We consider important questions of articles published in literature and this is favorably seen in the scientific world. We made some corrections that we considered relevant, which are described in the text, but decided not to value the comments in the brief communication that we consider irrelevant or when we noticed that the authors misinterpreted it, from the title and methodology used in our study, which was a retrospective study with limitations that were described and discussed in the original article ${ }^{2}$.

Two relevant points after a careful reading of the brief communication are that the article by these authors who used PENTO as a conservative treatment showed a serious error when they included bone necrosis of different etiopathogenesis in the same group ${ }^{4}$. Treatment with PENTO is one of the conservative treatment options as implicit in our study. Moreover, the literature considers good conservative treatment options for ORN and may dispense the need for major surgical treatment, which decreases morbidity and provides a better quality of life. Finally, a retrospective article that has limitations cannot be considered unreliable.

\section{ORCID}

Brena Rodrigues Manzano, https://orcid.org/0000-0003-

\section{$0412-2243$}

Paulo Sérgio da Silva Santos, https://orcid.org/0000-00020674-3759

\section{Authors' Contributions}

B.R.M. wrote and corrected the manuscript. P.S.S.S. wrote and revised the manuscript. All authors read and approved the final manuscript.

\section{Acknowledgements}

This study was financed in part by the Coordenação de Aperfeiçoamento de Pessoal de Nível Superior - Brasil (CAPES) - Finance Code 001.

\section{Conflict of Interest}

No potential conflict of interest relevant to this article was reported.

\section{References}

1. Seo MH, Kim SM. Essential review points on the retrospective study of osteoradionecrosis in the jaws. J Korean Assoc Oral Maxillofac Surg 2020;46:208-10. https://doi.org/10.5125/jkaoms.2020.46.3.208

2. Manzano BR, Santaella NG, Oliveira MA, Rubira CMF, Santos PSDS. Retrospective study of osteoradionecrosis in the jaws of patients with head and neck cancer. J Korean Assoc Oral Maxillofac Surg 2019;45:21-8. https://doi.org/10.5125/jkaoms.2019.45.1.21

3. Martos-Fernández M, Saez-Barba M, López-López J, EstrugoDevesa A, Balibrea-Del-Castillo JM, Bescós-Atín C. Pentoxifylline, tocopherol, and clodronate for the treatment of mandibular osteoradionecrosis: a systematic review. Oral Surg Oral Med Oral Pathol Oral Radiol 2018;125:431-9. https://doi.org/10.1016/ j.00oo.2018.02.004

4. Seo MH, Eo MY, Myoung H, Kim SM, Lee JH. The effects of pentoxifylline and tocopherol in jaw osteomyelitis. J Korean Assoc Oral Maxillofac Surg 2020;46:19-27. https://doi.org/10.5125/ jkaoms.2020.46.1.19

How to cite this article: Manzano BR, da Silva Santos PS. Reply to the brief communication "Essential review points on the retrospective study of osteoradionecrosis in the jaws". J Korean Assoc Oral Maxillofac Surg 2020;46:440-441. https://doi.org/10.5125/

jkaoms.2020.46.6.440 